\title{
A Multi-Port Optical Microfiber Coil Resonator
}

\author{
Rand Ismaeel", Timothy Lee, Feras Al-Saab, Yongmin Jung, and Gilberto Brambilla \\ Optoelectronics Research Centre, University of Southampton, Southampton, SO17 1BJ, UK \\ * Corresponding author: rmnilg10@orc.coton.ac.uk
}

\begin{abstract}
A new type of multi-port optical microfiber resonator is presented. The coupler-based coil resonator was fabricated by coiling a four port microfiber coupler around a low index support rod and then embedding the resulting device in a low refractive index polymer to improve its robustness. In this structure, light propagates along the coil whilst the beating between the supermodes of the coupler still present, giving an increased extinction ratio and an output spectrum strongly dependent on the microfiber coupler diameter.

OCIS codes: (140.4780) Optical resonators; (130.3990) Micro-optical devices; (060.1810) Buffers, couplers, routers, switches, and multiplexers.
\end{abstract}

Optical micro-resonators (loop, knot and coil) have gained a growing interest in a variety of fields due to their low absorption loss, fabrication simplicity, and easy coupling to the input/output fibers [1]. In particular, the microcoil resonator (MCR) exhibits interesting characteristics such as high quality (Q) factor, slow and fast light, robustness and 3D geometry [2]. However, many applications (like sensing or wavelength division mux/demux) require multi-port operation: various ideas have been suggested, such as the reef knot resonator (which is a four port version for the knot resonator) [3], the all-fiber add/drop filter [4] and the racetrack resonator (the four port version of the loop resonator) [5]. All these multi-port resonators have limited Q and poor long term stability. In this work, we present the multi-port microfiber coil resonator (MMCR) (shown in fig. 1), and discuss the fabrication and modeling of two MMCRs made from couplers with different diameters.

Microfiber couplers (MC) used to manufacture MMCRs were fabricated fusing two standard telecom optical fibres using the modified flame brushing technique [1]. The coupler minimum waist diameter was $3.2 \mu \mathrm{m}$ in MMCR1 and $0.7 \mu \mathrm{m}$ in MMCR2. MCs were then coiled around a support rod for 3 turns, using computer controlled rotation and linear stages [6]. In MMCR1, the pitch between the turns was approximately $2.5 \mu \mathrm{m}$.

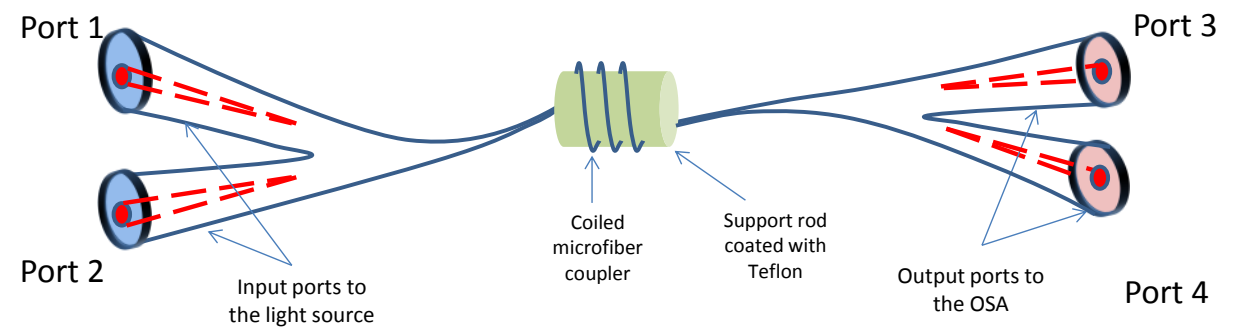

Fig. 1. Schematic of the multi-port microcoil resonator (MMCR): two microfibers are fused together at high temperature and coiled around a support rod of predetermined diameter. In the experiments, ports 1 and 2 are the inputs whilst, ports 3 and 4 are the outputs.

Fig. 2 shows the MMCR spectral properties, which are considerably different between the two devices. The output of MMCR1 (fig. 2(a)) shows three main characteristics: (1) a complimentary behavior between the spectra of the output ports, (2) a significantly stronger extinction ratio (compared to MCRs) and (3) also secondary resonance peaks. On the other hand, MMCR2 (fig. 2(b)) outputs are similar and resemble that of a MCR. This phenomenon can be ascribed to the smaller coupler diameter, which supports only the fundamental mode, hence the MMCR performs similarly to an ordinary MCR [2]; the short frequency modulation is possibly due to residual coupling in the output transition regions of the coupler.

The complementary behavior observed in the MMCR1 outputs could be explained by the presence, in the MC waist region, of more than one supermode which then beat and provide power exchange at the MC output arms. The maximum exchange occurs at the MCR resonant wavelengths, when the optical path is considerably longer than the physical path, allowing for a cumulatively larger amount of power to be exchanged than when off-resonance. For this reason, the maxima and minima of the two port outputs 
coincide. While resonances in an ordinary MCR result from light being trapped inside the resonator, in an MMCR, the additional interaction between supermodes take place, with the overall result of an increased extinction ratio.
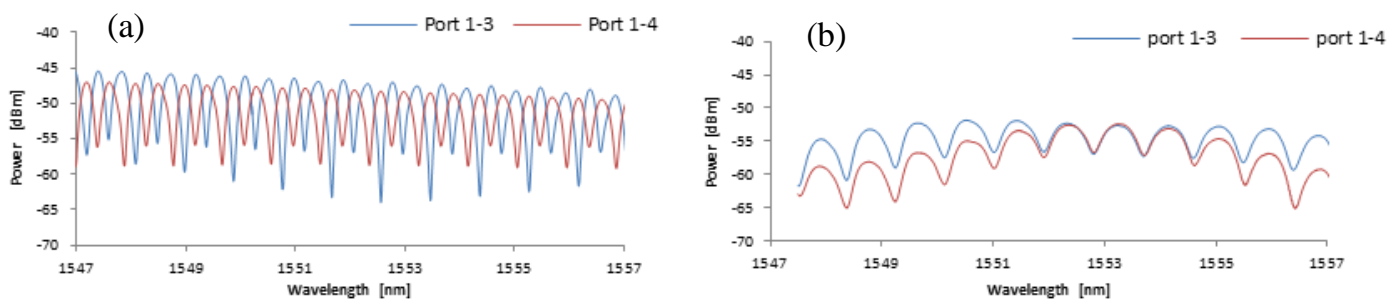

Fig. 2. The output spectra of (a) MMCR1, and (b) MMCR2. The reciprocal features between ports 1 -

3 and 1-4 typical of the coupler are visible in MMCR1 while they disappear in MMCR2.

MMCR was modelled by modifying the original MCR differential equations [2] to incorporate the coupler behaviour. Eqns. (1-2) describe the evolution of the electric field amplitudes at a distance along the turn which has a total length $L$. The amplitudes for each coupler arm are treated individually and the pitch is assumed to be sufficiently large that only coupling between neighbouring turns/arms is significant.

The MMCR transmission is therefore given by:

Where represents the propagation constant, (

), is the wavelength and

coupling coeficient $\kappa_{1}$ between modes inside of the coupler was numericall index. The coupling coefficient $\mathrm{K}_{1}$ between modes inside of the coupler was numerically calculated ( from the coupler cross section shown in fig. 3(a), while (which is the coupling coefficient of the light between the turns of the coil) was taken to be [2]. The simulated results in fig. 3(b) confirm the complementary behavior of the device as well as additional resonance peaks for the larger MC diameter.
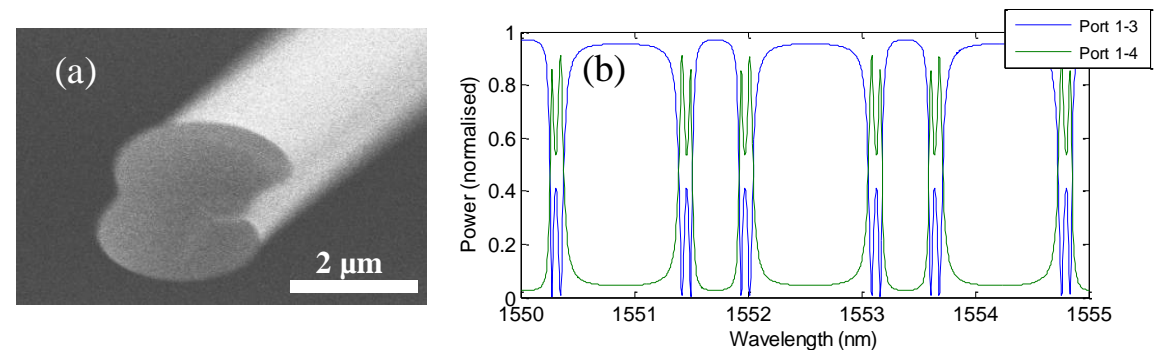

Fig. 3. (a) Scanning electron microscope image of the coupler dumbbell cross section used to calculate . (b) Simulated transmission of a 3 turn MMCR with $=36144.5 \mathrm{~m}^{-1}=5600 \mathrm{~m}^{-1}$. Coil diameter and propagation loss were assumed to be $1 \mathrm{~mm}$ and $4.6 \mathrm{~m}^{-1}$ respectively.

In conclusion, multi-port coil resonators where fabricated with different coupler diameters. For coupler diameters of a few microns, the effects of the modes beating inside the coupler are present in the MMCR transmission, while at smaller diameters $<1 \mu \mathrm{m}$ these effects vanish and the transmission of the MMCR is similar to that of the MCR.

\section{References}

[1] G. Brambilla et al., "Optical fiber nanowires and microwires: fabrication and applications", Adv. Opt. Photon. 1, 107-161 (2009).

[2] M. Sumetsky, "Optical fiber microcoil resonators," Opt. Express 12, 2303-2316 (2004).

[3] G. Vienne et al., "Demonstration of a reef knot microfiber resonator," Opt. Express 17, 6224-6229 (2009).

[4] X. Jiang et al., "All-fiber add-drop filters based on microfiber knot resonators,"Appl. Phys. Lett. 88, 223501, (2006)

[5] Y. Jung, G. Brambilla, G. S. Murugan, and D. Richardson, "Optical racetrack ring-resonator based on two U-bent microfibers," Appl. Phys. Lett. 98, 021109 (2011).

[6] Y. Jung, G. S. Murugan, G. Brambilla, and D. Richardson, "Embedded Optical Microfiber Coil Resonator with Enhanced HighQ,” IEEE Photon. Tech. Lett. 22, 1638-1640 (2010). 